\title{
Elena Carletti \\ Il chiarore che deforma \\ Processi deformanti nella poetica di Amelia Rosselli
}

\begin{abstract}
La poesia di Amelia Rosselli si rivela di stampo profondamente fenomenologico e prende le mosse da una realtà tumultuosa, in costante divenire. Il caos della realtà esterna viene interiorizzato in un processo di filtrazione deformante, indispensabile per raggiungere uno stato di appropriazione del reale.

Il presente saggio indaga da principio le implicazioni fenomenologiche nella poetica rosselliana, in linea con simili approcci sia in Italia, nelle teorizzazioni del Gruppo 63, che all'estero, nel saggio di Charles Olson. L'arte perde una volta per tutte il suo statuto di veridicità e diventa piuttosto un processo in fieri, un cauto accostamento al reale. In particolare, nella ricerca rosselliana il meccanismo deformante diventa imprescindibile per interiorizzare e "domare" il mondo esterno. Il reale viene sottoposto a curvature e deformazioni spazio-temporali secondo le leggi delle geometrie non euclidee e delle trasformazioni topologiche. Il risultato ottenuto, seppure distorto, si rivela concettualmente equivalente alla materia di partenza. La rappresentazione deformante corrisponde dunque a un processo di filtrazione fenomenologico che restituisce una versione della realtà analoga, nonostante le sue forme siano stravolte.

Alcune brevi analisi testuali mostrano come la tensione deformante da una parte venga utilizzata per appropriarsi e superare definitivamente l'esperienza traumatica passata, dall'altra divenga strumento per inserirsi nel canone letterario patriarcale. Il padre si offre come figura emblematica di superamento: il padre biologico che origina il trauma infantile, ma anche i padri della tradizione letteraria (Rimbaud, Scipione, Dante, Montale) i cui versi vengono rubati e turbati, deformati in un discorso finalmente a misura di donna.
\end{abstract}

A più riprese Amelia Rosselli ha affermato di praticare una poesia profondamente realista. Tuttavia, a chiunque si sia imbattuto nella sua opera sarà senza dubbio capitato di rimanere disorientato da una simile pretesa. Il realismo rosselliano si spiega piuttosto come volontà di adesione, o meglio di "domazione" di un reale caotico e metamorfico percepito in un inarrestabile divenire.

Se il punto di partenza è il reale, per quanto sottoposto a una certa torsione nel testo letterario, occorre prima di tutto chiarire il rapporto che si instaura tra scrittore e realtà. Dagli anni Cinquanta prende a radicarsi la concezione di un fare poetico improntato ad un'attenta ricerca fenomenologica, anche a partire dalle esperienze di Charles Olson, filtrate nel panorama di ricerca letteraria italiana

The e-journal «altrelettere» is hosted at the URL: http://www.altrelettere.uzh.ch , in accordance with the Open Access Policy of the University of Zurich. Please cite this article as follows: Elena CARLETTI, Il chiarore che deforma - Processi deformanti nella poetica di Amelia Rosselli, in «altrelettere», 16.1.2015, DOI: 10.5903/al_uzh-27.

(C) This article is licensed under a Creative Commons Attribution 2.5. Switzerland (CC BY-NC-ND 2.5). Please read the license terms on the website: http://creativecommons.org/licenses/by-nc-nd/2.5/ch/deed.en 
soprattutto nel lavoro del Gruppo 63 e di Amelia Rosselli. Un simile approccio, se da una parte si profilava come risposta a quelle rivoluzioni artistiche primonovecentesche che avevano irrevocabilmente posto in crisi il concetto tradizionale di rappresentazione, dall'altra costituiva un discorso dialogico con il complesso di innovazioni tecnico-scientifiche che avevano trasformato il volto di un secolo. Per dirla con Umberto Eco:

[si tratta della] reazione dell'arte e degli artisti (delle strutture formali e dei programmi poetici che vi presiedono) di fronte alla provocazione del Caso, dell'Indeterminato, del Probabile, dell'Ambiguo, del Plurivalente; la reazione, quindi, della sensibilità contemporanea in risposta alle suggestioni della matematica, della biologia, della fisica, della psicologia, della logica e del nuovo orizzonte epistemologico che queste scienze hanno aperto. [...] l'arte contemporanea si trova a fare i conti con il Disordine. Che non è il disordine cieco e insanabile, lo scacco di ogni possibilità ordinatrice, ma il disordine fecondo di cui la cultura moderna ci ha mostrato la positività; la rottura di un ordine tradizionale, che l'uomo occidentale credeva immutabile e definitivo e identificava con la struttura oggettiva del mondo. (ECO 2006, 2)

Protagonista è dunque un «disordine fecondo» che viene a coincidere, alla luce delle recenti ricerche fenomenologiche in campo filosofico (Husserl, MerleauPonty; in Italia Banfi e Paci), con una realtà scossa, ricomposta, deflagrata: una realtà che si offre come imprescindibile punto di partenza per la costituzione del processo artistico. ${ }^{1}$ La particolarità della nuova produzione poetica nascente nel dopoguerra si trova, dunque, in una sorta di slittamento nell'approccio al reale: l'arte, considerata nella sua cosalità uno strumento - o medium - di indagine, incarna in questi anni un processo in divenire che mette in discussione gli apparati estetici tradizionali per privilegiare una visione problematica e complessa del fare artistico. Di fatto l'arte non avanza più alcuna pretesa di incarnare il vero, e viene a definirsi, semmai, come un tentativo di avvicinamento al reale. Proprio Amelia Rosselli avverte che se ancora è ammissibile parlare di aureola poetica, questa deve essere conferita alla realtà stessa: a distanza di un secolo un avvenimento accidentale, la caduta dell'aureola baudelairiana nel fango, viene tramutato in gesto intenzionale, in consapevole incoronazione del mondo: «L'alba si presentò sbracciata e impudica; io / La cinsi 
di alloro da poeta: ella si risvegliò / Lattante, latitante» (Rosselli 2009, 230; corsivo mio). Il poeta decide di privarsi dell'alloro per poter incoronare l'«alba», incarnazione metonimica di una realtà inedita che non può più essere giudicata $a$ priori e che assurge a vera protagonista del fare poetico. Il gioco paronomastico lattante - latitante rimarca la volontà di entrare in rapporto con un'entità che è alle sue prime comparse («L'alba si presentò»), e quindi nascente, in fasce. $\mathrm{Si}$ rientra, così, nella dinamica prima accennata di avvicinamento a un reale insondato e sfuggente, che fa coincidere la ricerca fenomenologica con il processo artistico stesso.

La poesia, di conseguenza, abbandona una volta per tutte ogni sentenziosità e diventa domanda, tentativo, azione. Nel 1965 Amelia Rosselli, Piero Dorazio, Vittorio Gelmetti, Pietro Grossi e Giordano Falzoni partecipano a un dibattitto in cui musica, poesia e pittura confrontano i propri procedimenti artistici: durante la conversazione gli artisti si interrogano sulle intenzioni alla base delle proprie opere, in particolare Dorazio afferma: «Il problema è nel fare, e la soluzione la cerco facendo un quadro, quindi pongo il problema e cerco la soluzione allo stesso tempo» (CAPUTO 2004, 36). Non è un caso che proprio fare diventi parola chiave anche nel discorso della poesia sperimentalista, non solo nel suo riflesso etimologico (poiesis), ma in quanto concetto programmatico, avanzato da Alfredo Giuliani nellintroduzione alla ormai canonica antologia dei Novissimi: «quel fare, che, come abbiamo detto, sentiamo coincidere con il "contenuto"» (GIULIANI 1961, XVIII; corsivo dell'autore).

L'impronta complessa e fenomenologica della poesia si costruisce, lo abbiamo accennato, sull'eco di discorsi letterari internazionali: di grande influenza, non solo per la ricerca di Amelia Rosselli, è il pensiero elaborato nel 1950 da Charles Olson nel saggio Projective Verse, ${ }^{2}$ per cui il meccanismo propulsore del verso viene alimentato dalla registrazione di una percezione dinamica e in costante divenire, sottomessa a una certa urgenza della parola:

ONE PERCEPTION MUST IMMEDIATELY AND DIRECTLY LEAD TO A FURTHER PERCEPTION (...) [it] is a matter of, at all points (even, I should say, of our management of daily reality as of the daily work) get on with it, keep moving, keep in, speed, the nerves, their speed, the perceptions, theirs, the acts, the split second acts, 
the whole business, keep it moving as fast as you can. (OLSON 1966 [1950], 2; corsivo mio)

È ancora Alfredo Giuliani a citare esplicitamente il saggio di Olson in appendice ai Novissimi per affermare una comunanza di intenti che spazia dalla dimensione fenomenologica del testo poetico, alle tematiche concettuali (la riduzione dell'io) e metriche (il verso «dinamico»). 3

La questione poetica del rapporto con la realtà - e l'appropriazione del presente - lungi dall'imporsi come soluzione univoca viene declinata in modo specifico da ogni autore, restituendo, però, una visione comune, battezzata da Giuliani «schizomorfa»:4 l'esperienza viene osservata, spintonata, capovolta e in qualche modo distorta nel processo di filtrazione poetica. Si noti come nel passaggio seguente di Giuliani anche il «problema di realismo» venga rivisitato e risponda piuttosto a un meccanismo di contrazione della realtà esperita, quindi a un processo deformante.

Pensiamo che parlando di noi o d'altro o di niente (de dreit nien), la poesia debba aprirci un varco: nel rispecchiare la realtà rispondere al nostro bisogno di attraversare lo specchio. Dei poeti qui raccolti mi sembra che il solo Pagliarani si sia fatto un problema di «realismo» letterario, ma sempre contrando la realtà sperimentata, mai credendolo un contenuto di per sé sufficiente a rinnovare la poesia. Tutti noialtri, ci siamo fatti un problema di verità, di rinnovamento strutturale, non di realismo coatto. (GIULIANI 1961, XIV; corsivo dell'autore)

Nonostante la Rosselli abbia ribadito a più riprese la propria indipendenza dal Gruppo 63, un substrato comune, per altro di derivazione preponderantemente filosofica, aveva iniziato a trasfondersi nella ricerca poetica di quegli anni (a partire, lo si è visto, proprio da esperienze extraeuropee - il caso di Charles Olson). Come giustamente fa notare Niva Lorenzini5 il movimento della neoavanguardia, seppur rifiutato dalla poetessa come mera applicazione di un'etichetta, presenta più di un punto in comune con la ricerca rosselliana: un accostamento giustificato da quello sperimentalismo linguistico e dei mezzi che accomunava l’indagine poetica della Rosselli a quella del Gruppo 63.

Ecco allora che il realismo rosselliano si configura come un tentativo, nella sua più profonda sostanza "schizomorfo",6 di sintetizzare la realtà e l'esperienza 
attraverso il fare poetico. Nella poetica di Amelia Rosselli la volontà di sintesi e di "domazione" del reale si attua in un processo di forte devianza, deformazione, trasfigurazione. L'esperienza, o il mondo fuori, si riflette nel testo poetico per mezzo di uno specchio deformante, anamorfico, che rinuncia definitivamente alla linearità dello specchio tradizionale e, piuttosto, sottopone a continua torsione ogni datità esterna. Si tratta di un meccanismo di registrazione che interpone tra il soggetto e la realtà un filtro «attraverso il quale le cose, allargandosi in immagini surreali o allungandosi in forme allucinate, tornino a svelarsi» (BALESTRINI 2002, XIV), dirà con lampante incisività Angelo Guglielmi.7

La tensione deformante per arrivare al possesso del reale si rivela pietra angolare di tutta l'opera rosselliana: l'instancabile metamorfismo, infatti, non si limita alla traduzione mimetica della realtà esperita nei "contenuti” delle poesie, ma esonda dal testo fino a investire ogni livello della produzione poetica inglobando in primis il rapporto con la tradizione - sia rispetto alla metrica che al canone letterario - ma anche la lingua nella sua materialità.

\section{Poesia come spazio-tempo deformante}

Il testo poetico in cui Amelia Rosselli ingabbia la realtà che la circonda si configura come vero e proprio luogo, uno "spazio metrico" di misurazione che riporta determinate coordinate spazio-temporali. Sulla sua soglia avviene il processo di filtrazione deformante a cui faceva riferimento anche Guglielmi: l'autore si accosta alla realtà esperienziale e la trasfigura, la deforma, la tra-duce (trasporta) in un modello chiuso e finito che reca in sé la traccia del mondo universale nel suo dinamismo.

Il testo si configura dunque come un luogo "di secondo grado" e al suo interno viene ricreato un corrispettivo della realtà. Jurij Lotman, ne La struttura del testo poetico, getta nuova luce sulla definizione del rapporto tra il testo e il reale grazie alla scelta di applicare modelli geometrico-matematici all'indagine dello spazio testuale. Si tratta, lo vedremo, di un contributo particolarmente illuminante per comprendere il processo di "deformazione" messo in atto da Amelia Rosselli. Lotman descrive la relazione tra l'opera d'arte e il mondo esterno 
in questi termini: «Limitata nello spazio, l'opera d'arte diventa modello di un mondo senza confini» (LOTMAN 1990, 253). Essa è sempre "traduzione". Le due realtà sono dunque equivalenti (hanno, cioè, lo stesso valore). Lotman arriva a questa conclusione dopo aver paragonato il testo letterario alla geometria di Lobacevskij: la rivoluzione di questo tipo di geometria, rispetto alla tradizionale geometria euclidea, risiede in una diversa configurazione dello spazio. Se Euclide considera lo spazio infinito, Lobacevskij ragiona nei limiti di uno spazio chiuso, reso possibile dal concetto di curvatura dello spazio, che è però concettualmente equivalente a quello euclideo. Lo spazio di Lobacevskij, come il testo letterario, è dunque un luogo chiuso che corrisponde all'infinito del fuori.

Non desterà troppa meraviglia scoprire che Amelia Rosselli era al corrente di certe teorie geometrico-matematiche. La poetessa che afferma «La scienza dei numeri era la mia fortitudine» (Rosselli 2009, 205), e che intitola un saggio (Spazi Metrici) e una raccolta (Serie Ospedaliera) ${ }^{8}$ utilizzando termini matematici, annota nel giovanile Diario in Tre Lingue:

Courant \& Robins

what is mathematics

La geometria analitica

see music, see Mathm. see Aut Aut, see Gibbeon

(RossELLI 2009, 103)

In Che cos'è la matematica? di Courant e Robbins la geometria analitica viene descritta come «un metodo che ha prodotto un profondo mutamento nella scienza matematica, approdando a una unificazione della geometria, dell'analisi e dell'algebra in un sistema organico» (COURANT e RoBBins 1964, 259). Nel testo di Courant e Robbins essa viene studiata in relazione alle possibili trasformazioni delle figure, dette trasformazioni topologiche: queste deformazioni restituiscono forme che sono topologicamente equivalenti, in quanto mantengono determinate proprietà.

Gli esempi più intuitivi di trasformazioni topologiche in generale sono le deformazioni. Supponiamo che una figura, come una sfera o un triangolo, sia costituita da un sottile foglio di gomma o sia disegnata su di esso, e che poi si tenda, e contorca il foglio in tutti i modi possibili $[. .$.$] La posizione finale di una figura sarà$ 
allora un'immagine topologica dell'originale. Un triangolo può essere deformato in un altro triangolo qualsiasi, o in un cerchio, o in un'ellisse, e quindi queste figure hanno esattamente le stesse proprietà topologiche. (COURANT e ROBBINS 1964, 363)

Siamo all'interno del concetto di realismo da cui è partita questa riflessione: a una deformazione di materia corrisponde una forma finale differente, eppure equivalente, compressa in uno spazio finito: «lo spazio non era infinito, bensì prestabilito, come se comprimesse l'idea o l'esperienza o il ricordo» (RossELLI 2009, 341), scriverà la Rosselli in Spazi Metrici. Attraverso queste deformazioni si accalappia e soggioga la percezione della realtà che viene dunque trasposta nella dimensione del testo poetico, modello, questo, di una realtà universale. La verosimiglianza lascia posto all'equivalenza: le linee rette vengono sistematicamente curvate, ma rimangono pur sempre linee.

My Clothes to the Wind e A Birth sono i testi che meglio illustrano questo meccanismo deformante. Le due prose inglesi fanno parte di un percorso propedeutico e formativo che la Rosselli intraprende per liberarsi dal fardello della nevrosi personale e raggiungere piena maturazione letteraria. L'autrice stessa ne parla come di un'esperienza preparatoria, volta a inaugurare la produzione poetica vera e propria: «Primi Scritti 1952-1963 fu comprato da Guanda nell'8o e son tutte cose scritte in prosa o poesia, preparatorie al pubblicare il primo libro nel '64, in italiano. [...] Comunque questo è un libro preparatorio giovanile.» (VENTURINI e DE MARCH 2010, 325). Tentativi analoghi erano stati fatti nei primi testi in assoluto scritti dall'autrice, Phantasy end of November e Phantasy beginning of December, frutto di un'autoanalisi compiuta utilizzando la tecnica dell'immaginazione attiva. 9 Sulla necessità di risolvere i propri problemi psichici per potersi dedicare alla letteratura in modo responsabile, l'autrice inoltre scrive:

[S]ono del parere che non si è scrittori se non si è fatta una analisi personale, per levare di mezzo i problemi più urgenti che possono intralciare una visione del mondo un pochino obiettiva e utile agli altri. Se no, si scrive una poesia ad uso personale. Molti dicono che scrivono per risolvere le loro nevrosi. Scusi, ma tutti siamo nevrotici dice Freud, a che pro? Vai dall'analista otto mesi e poi scrivi il capolavoro. Almeno cerca di risolvere i problemi più brucianti che ti impediscono di scrivere, che ti impediscono di entrare nell'inconscio, di rompere la barriera tra conscio e inconscio. 
Almeno risolvi quelli brutalmente ovvi. Un mare di bella poesia qualche volta e dei bei romanzi, escono solo perché vogliamo risolvere i nostri personali problemi, o di famiglia, confessandoli. Meglio parlarne con uno psicologo per poter scrivere poi di cose più generali e più utilmente. Il ritorno al privato ha quel difetto, che non prende in considerazione l'egoismo assoluto. Noi pretendiamo di dare qualcosa ad un pubblico che poi è di uso privato, un'autoanalisi confusa e impasticciata. Meglio un analista da giovane non soltanto per risolvere in parte la propria nevrosi - nessuno la risolve del tutto, ma per non dare in pasto ai leoni i fattacci nostri e farli passare per arte. L'arte supera la problematica del trauma o dovrebbe farlo almeno in parte. (DE MARCH e VENTURINI 2010, 239).

Benché la Rosselli, forse troppo umilmente, neghi uno statuto pienamente letterario a questi scritti (considerandoli più che altro materiale di preparazione), il meccanismo di rappresentazione deformante qui messo in atto rimarrà alla base della poetica successiva. In questi testi l'io, prima della sua tentata riduzione a favore di un io-noi universale, è intento a affrontare il trauma delle proprie personali nevrosi. L'io ricopre allo stesso tempo il ruolo di autore (nelle vesti di un Dio creatore) e personaggio (soggetto alla fragilità mortifera) e opera una deformazione di un passato allucinato, fortemente autobiografico, allo scopo di "domarlo" e liberarsene una volta per tutte. Il tentativo di scrittura corrisponde a un tentativo di risanamento del Sé frammentario in cui si evidenzia una modalità di percezione che segue i meccanismi dell'inconscio, frutto di un percorso d'identificazione psicanalitico di matrice ancora junghiana basato sull'immaginazione attiva. Il passato, quindi la realtà esperita, viene rievocato e rivissuto in una dimensione psicolinguistica spazio-temporale, dove gli elementi di spazialità e temporalità rispondono a un processo di rappresentazione deformante.

My Clothes to the Wind mette in scena una spazialità esplosa: i personaggi sono introdotti attraverso scorci d'intensità violenta, fanno brevi comparse e si rigettano in uno spazio letterario deformato, curvo, in cui si accampano strade contorte, campagne deterritorializzate, la città di Londra e persino corridoi dalle architetture assurde, affacciati su fiumi. Ogni linearità è rotta o deforme e nega una rappresentazione verosimigliante della realtà descritta. In $A$ Birth la deformazione dello spazio si estende programmaticamente alla dimensione temporale: l'autrice si appresta a compiere un viaggio a ritroso nello spazio- 
tempo, fino a risalire alla nascita. Gli spazi che incontra sono incerti, stravolti, sistematicamente curvi: il cielo è «round», la torre «curved», la strada «crooked» (Rosselli 2009, 125). Anche in questo caso la spazialità si offre nella sua forma complessa; non si tratta di una superficie lineare ma di uno «space imbedded in space» (RossELLI 2009, 127).

La Rosselli sembra distorcere la realtà del ricordo proprio secondo le leggi delle geometrie non euclidee, per cui ogni forma può essere deformata in una sfera (COURANT e RoBbins 1964). Viene in questo modo ripreso il concetto di circolarità, trait d'union tra razionalità e inconscio: gli elementi geometrici e matematici alla base della formulazione metrica trovano un corrispettivo nel «rullo cinese» tradotto in «delirante corso di pensiero occidentale» (RossELLI 2009, 338; in Spazi Metrici), per cui l'Occidente razionale e schizofrenico cerca di conciliarsi all'Oriente della ciclicità Zen, dell'Ouroboros, del Mandala.

La deformazione del reale porta infine a una presa di possesso: attraverso la curvatura si impedisce la fuga del ricordo e si stabilisce una "curva appartenenza" - «Curve the triangle out of escape and the landscape into curved belonging» (Rosselli 2009, 125; corsivo mio) -, che determina un'acquisizione epifanica: entrambe le prose si chiudono, infatti, con la parola «recognition», quindi riconoscimento, presa di coscienza. La "curvatura" delle forme lineari e dei paesaggi si prospetta come artificio necessario per poter assimilare il reale.

La deformazione diventa dunque un metodo di “domazione" della realtà caotica, in grado di ristabilire equilibrio. Nella poesia riportata di seguito Amelia Rosselli si cala nelle vesti di un Cristo deformante (che vedremo essere simbolo di una tentata riconciliazione di opposti), in una variante dell'identità io-dio

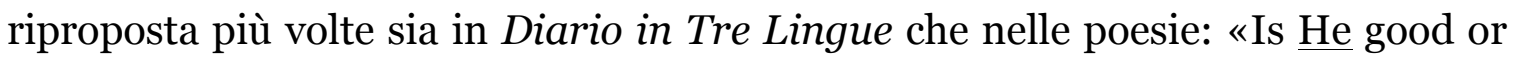
am I god?» (Rosselli 2009, 90; Diario in Tre Lingue), «Perché Iddio (Io) mi perdonasse» (ROSSELLI 2009, 250). L'accostamento dell'io alla figura divina (sia trinitaria: Padre, Figlio, Spirito Santo, ma anche divinità pagana) è una scelta che intende annullare le antinomie nell'unità: tra autore-creatore e personaggiocreato, tra uomo e donna e, non meno importante, tra padre e figlia. ${ }^{10}$ 
Si tratta quindi di una sovrapposizione complessa: il Cristo dagli «occhi Bizantini splendenti e crudeli», eco degli occhi «celestiali» e «obliqui» di un altro componimento, tutto il mondo è vedovo, (Rosselli 2009, 333), è l'autrice-dio che sottopone il mondo a deformazione - «Il Cristo deformava / il mondo in mille maniere, catacombe delle lacrime» - in un percorso che, seppur pervaso dal lutto, mette in atto un ciclo di morte e resurrezione: «curva di spalla sepolta e rinata». La passione di Cristo, la croce informe e poi quadriforme, si identifica nella parola del Cristo-autore, «Cristo trainava la sua croce» - «parole trainanti nella polvere» - «forma cunea, alfabeto - triangolo», che attraverso il suo potere metamorfico permette l'alternarsi di distruzione e rigenerazione in un movimento conciliatorio di opposti, in cui anche terra e cielo si incontrano.

Il Cristo trainava (sotto della sua ombrella) (la sua croce) un informe materiale; parole trainanti nella polvere del dipinto del chiostro di vetro. Sotto alla sua chiostra di vetro il Cristo trainava una sciabola. Dodici pecore sogghignavano distrattamente alla sua predica. Io montavo in arabeschi il mio pudore dozzinale, su per le vetrate ricurve della sua sala da pranzo, margherite colate in piombo su dei prati e i cieli oscuranti di blu feroce. Io salivo i gradini della pietà molto ben concentrata in se stessa, con la croce quadriforme della sua durezza alle spalle. Il Cristo incrociato era una colomba, che spaziava teneramente, lusingava con la sua coda i teneri colori del cielo appena accennato. Il Cristo deformava il mondo in mille maniere, catacombe delle lacrime. I suoi occhi Bizantini splendenti e crudeli stagliavano rondinelle nel cavo del cuore. La crudeltà si taceva forse meno maestra del mondo, o universo con la sottana troppo piccola, se lui piangeva. Io che cado supina dalla croce m'investo della sua mantella di fasto originario. Bellezza armonia che scintilli anche per i prati ora secchiti: marmo che non cade, curva di spalla sepolta e rinata, con la spala che intacca i geroglifici del mondo. Forma cunea, alfabeto - triangolo, - punta al cielo le tue dita sporcate di terriccio.

(ROSSELLI 2009, 273)

Il filtro poetico deformante viene impiegato dunque come strumento di "domazione" della passata esperienza; filtro che ha il potere, lo si è accennato poco fa, di conciliare gli opposti e restituire unità al sé frammentario. La 
deformazione non si limita alla dimensione dell'esperienza prettamente biografica, ma investe tutti i livelli della percezione, incluse l'esperienza ricettiva dell'io-lettore e quella creativa dell'io-autore. In particolare, nel passaggio dalla fase di ricezione a quella di creazione, la Rosselli mette in atto un'opera di invasione dei testi del canone patriarcale per sottoporli a deformazione e dunque per renderli propri, in un processo analogo a quello della revisione mitologica femminista, qui nella definizione di Alicia Ostriker:

Let me at this point therefore define the term "revisionist mythmaking" [...] Whenever a poet employs a figure or story previously accepted and defined by a culture, the poet is using myth, and the potential is always present that the use will be revisionist: the figure or tale will be appropriated for altered ends, the old vessel filled with new wine, initially satisfying the thirst of the individual poet but ultimately making cultural change possible. (SHOWALTER 1986, 317; corsivo mio)

La revisione operata sui testi della tradizione implica una distorsione riscontrabile in primis sulla lingua: ancora una volta è lo stravolgimento metamorfico a determinare una presa di possesso e, in questo caso, un posizionamento attivo rispetto alla tradizione.

I meccanismi di distorsione, già sperimentati nei primi tentativi di scrittura, si ripresentano con ulteriore consapevolezza nel poemetto La libellula, del 1958.11

Fluisce tra me e te nel subacqueo un chiarore che deforma, un chiarore che deforma ogni passata esperienza e la distorce in un fraseggiare mobile (CORTELlESSA 2007, 12)

Nell' «inventata invettiva» dai caratteri bellicosi ${ }^{12}$ gli opposti si scontrano con spiccata violenza per incontrarsi di nuovo, fondersi, trasfondersi in una perdita di confini precisi o di identità univoche. Tutto procede per metamorfosi: il lettore si trasforma in autore, il figlio in genitore, la donna in uomo, l'io e il tu terminano in una fusione promiscua, androgina e complementare.

Molto è stato detto sulla Libellula come panegirico femminista, «largo esperimento» identitario di un io-scrivente femminile che tenta di affermarsi all'interno di una patriarcale «santità dei santi padri». In Italia più che all'estero le conquiste socioculturali delle donne tardano ad arrivare: nel 1958 si registra 
ancora una completa e imbarazzante assenza di autori donne nel canone letterario italiano,13 quando già nel 1929 Virginia Woolf pubblicava il suo provocatorio e "canonico" Una stanza tutta per sé. In Italia sarà proprio Amelia Rosselli, per altro soltanto nel 1978, a irrompere nell'antologia di Mengaldo, Poeti italiani del Novecento, e quindi a incrinare definitivamente lo status quo del patriarcato letterario.

Nel 1958, tuttavia, l'operazione deve essere ancora compiuta e Amelia Rosselli, ben consapevole della sua condizione socio-letteraria, si trova a dover scrivere nella tramandata ossessione di una lingua forgiata a misura di uomo. Lei stessa, nell'antologia Donne in poesia del 1976, curata da Biancamaria Frabotta, dirà:

Che esista una differenziazione linguistica femminile è da sospettarsi, sia essa da quarto mondo o biologica, addirittura in origine, anche se rimane da studiarsi in campo sperimentale, secondo me. Che la donna imiti l'uomo nello scrivere è ovvio e forse un suo rintracciare forme artistiche sue originarie o perfino chimicamente più sue, resta problema aperto. Ma se la donna ne è conscia, il suo scrivere si fa davvero più laborioso! [...] Se il movimento femminista si propone una piattaforma sociopolitica sono anche d'accordo, ma resta da studiare un'altra piattaforma forse, quella appunto biologico-culturale (o perfino mistica). (FRABOTTA 1976, 47)

Come fare, allora, a risolvere il «problema aperto» e appropriarsi della lingua dell'uomo adeguandola a un discorso «biologico-culturale» femminile? Se la parola appartiene all'altro-da-sé è necessario compiere un furto calcolato. La seguente dichiarazione di Michail Bachtin, tratta da Estetica e romanzo, può a buon ragione essere assunta come premessa metodologica:

La parola della lingua è semi-altrui. Essa diventa "propria", quando il parlante vi installa la propria intenzione, il proprio accento, quando padroneggia la parola e la associa alla propria aspirazione semantica e espressiva. Prima di questo momento di appropriazione la parola non è nella lingua neutra e impersonale, ma sulle labbra altrui, negli altrui contesti, al servizio delle altrui intenzioni: di qui essa deve essere presa e resa propria. (BACHTIN 1997, 107)

La parola «deve essere presa e resa propria». Proprio dalla constatazione che la lingua appartiene ad un altro, infatti, si genera la necessità del furto, a maggior ragione per una scrittrice biograficamente priva di una lingua madre, che sceglie la lingua del padre per esprimersi in senso sociale e letterario: «la mia lingua 
cosiddetta materna è paterna, perché ho preso molto da mio padre, ho vissuto molto più a lungo in Italia...» (DE MARCH e VenturinI 2010, 267), «Però ho dovuto insegnarmi l'italiano; per istinto l'ho fatto sui libri di poesia, strano a dirsi» (DE MARCh e VENTURINI 2010, 277). L'italiano viene scelto in quanto lingua del padre e appreso proprio attraverso la parola dei padri letterari. Emanciparsi dal dominio dell'altro e destrutturare il fallologocentrismo implicito nel canone patriarcale richiede dunque uno scacco alla legge lacaniana del Padre operato dal suo interno. L'impossessarsi del discorso, come ci ricorda ancora Alice Ostriker, costituisce un atto necessario di ribellione al Padre, volto alla liberazione della donna «invisibile» o «silenziosa», altrimenti confinata nelle stanze del manicomio:

Though the language we speak and write has been an encoding of male privilege, what Adrienne Rich calls an "oppressor's language" inadequate to describe or express women's experience, a "Law of the Father" which transforms the daughter to "the invisible woman in the asylum corridor" or the "silent woman" without access to authoritative expression, we must also have it in our power to seize speech and make it say what we mean. (SHOWALTER, 1986, 315; corsivo mio)

Esattamente questo accade ne La Libellula rosselliana, dove l'io-donna ruba la parola al tu-uomo e vi installa la propria personalissima intenzione, dando luogo al suo interno ribaltamento. La sacralità intoccabile della tradizione è ora passibile di profanazione, dopo l'avvenuto "salto per un addio più difficile» (CORTELLESSA 2007, 1). Il verso maschile viene quindi sottoposto a torsione fisica della materialità sonora, viene deformato in un «fraseggiare mobile, / distorto, inesperto, espertissimo linguaggio / dell'adolescenza!». Un verso «inesperto» perché inedito, nuovo, giovanile (l'adolescente che afferma la sua prima identità) eppure «espertissimo», perché consapevole, necessario, liberatorio. ${ }^{14}$

Dante, Campana, Montale, Rimbaud, Scipione vengono fagocitati nel discorso poetico rosselliano, in una inumazione assimilante che ricalca quella della mantide assassina: «io avanzo il pugnale in un pugno stretto, / e ti ammazzo» (CORTELLESSA 2007, 5). La deformazione avviene nella parola prima rubata e poi sistematicamente turbata in più ritorni, all'interno di un sistema variantistico che si impianta sull'idea del ritornello e quindi di una struttura ossessivante. Si veda 
ad esempio il dantesco «Nel mezzo del cammin di nostra vita» deformato e torto in: «Nel mezzo di una luce che è / chiara e di un'altra che è la cattiveria in persona / cerco il ritornello. Nel mezzo d'un gracile cammino / [...] io cerco, e tu ti muori presso / un albero infruttuoso, sterile come la tua mano» (CoRTELLESSA 2007, 2). Lo stesso vale per la chimera di Campana, l'Esterina di Montale, l'Ortensia di Rimbaud di cui si distorce non solo il verso linguistico originale, ma anche il personaggio femminile riscritto e rimitizzato. Seppure a scopo dimostrativo occorrerebbe analizzare qui le ulteriori "deformazioni versali", mi pare più opportuno rimandare a studi già dedicati alle intertestualità della Libellula, tra i quali quelli di Annovi, Lo Russo, Carbognin. ${ }^{15}$

La lettrice, ribaltatasi ora in autrice, parla a un lettore ancora ignoto: «Ricorda questa mia vita attaccata / all'ignoto sorseggiare delle tue pupille» (CORTELLESSA 2007, 6), e non sa se il suo «grido» raggiunga la destinazione, «non so se tu lo sai che io rimo interamente per te» (CORTELLESSA 2007, 6), «non so se questo grido ti raggiunga» (CORTELLESSA 2007, 7).

L'oggetto passivo femminile può finalmente trasformare se stesso in soggetto attivo e a sua volta passivizzare la figura maschile: si pensi all'esemplare ribaltamento del «deo dalle fulgide chiome» e all'effetto quasi grottesco provocato dal capovolgimento dei ruoli. ${ }^{16}$ L'operazione di rovesciamento è tanto più necessaria se si intende far vacillare un sistema patriarcale costruito in termini di economia binaria, entro il quale la donna è portata ad assumere un ruolo secondario, passivo e quindi oggettuale. Adriana Cavarero ne parla in questi termini:

L'economia binaria si fonda appunto su una logica bipolare che, a partire dalla positività del polo maschile, decide la negatività di quello femminile. Posto l'uomo come soggetto, la donna risulta perciò oggetto; posto il primo come il sé, la seconda risulta l'Altro. (CAVARERO 2002, 84)

A intuire la natura subordinata e "altra" della donna era stata già Simone De Beauvoir ne Il secondo sesso, dove scrive:

Ora, la situazione della donna si presenta in questa singolarissima prospettiva: pur essendo come ogni individuo umano una libertà autonoma, ella si scopre e si sceglie 
in un mondo in cui gli uomini le impongono di assumere la parte dell'Altro; in altre parole, pretendono di irrigidirla in una funzione di oggetto e di votarla all'immanenza perché la sua trascendenza deve essere perpetuamente trascesa da un'altra coscienza essenziale e sovrana. Il dramma della donna consiste nel conflitto tra la rivendicazione fondamentale di ogni soggetto che si pone sempre come essenziale e le esigenze di una situazione che fa di lei un inessenziale. (DE BEAUVOIR 1984, 27)

Virginia Woolf, da parte sua, denuncia la condizione di subordinazione oggettuale della donna anche all'interno del patrimonio canonico e letterario della tradizione. In Una stanza tutta per sé l'autrice, interrogandosi sul rapporto donna-letteratura, descrive come all'interno del British Museum figurassero centinaia di libri scritti da uomini sulle donne (quindi da soggetti maschili che si pronunciano su oggetti femminili), mentre erano del tutto assenti libri scritti da autrici sugli uomini. ${ }^{17}$ Una donna che si formi su una letteratura connotata in questi termini, sicuramente parziale, sarà portata a identificarsi implicitamente con la voce narrante dell'uomo e, di conseguenza, a decodificare la figura femminile come oggetto passibile di possesso.

La componente maschile all'interno dello sguardo femminile, quindi, è il risultato di una inalienabile e secolare tradizione culturale, pur passibile di contestazione. Se l'io-donna si ritrae adottando un punto di vista maschile questo avviene perché maschile è la rappresentazione sociale, artistica, letteraria che le è stata tramandata nel tempo: la donna, inevitabilmente, si guarda allo specchio con occhi di uomo, ${ }^{18}$ in lei confluiscono due contrastanti visioni. Anche per questo motivo l'elemento maschile, nell'opera di Amelia Rosselli, risulta bellicosamente integrato nella rappresentazione del sé androgino.

La scelta dell'androginia, dunque, risponde a una volontà di pacificazione delle tensioni belliche sottese all'incontro-scontro degli opposti, per arrivare a un ricongiungimento complementare. L'androgino si profila come alter ego testuale onnivoro, in grado di fagocitare e comporsi di elementi eterogenei: in questo modo l'io biografico viene sottoposto a una riduzione di tipo neoavanguardistico, rendendo possibile la costruzione di un'identità universale che non si limita a mescolare tratti maschili e femminili, ma che include ogni tipo di opposizione fino a culminare nella scomparsa dell'univocità: «esiste / un solo io, esiste un solo tu» (CORTELLESSA 2007, 12). L’economia binaria della critica femminista, 
così come la coppia animus-anima junghiana, non sono che elementi di partenza verso un androginia più ampia dai tratti mistici.

Il raggiungimento di uno stato di compresenza degli opposti è reso possibile, ancora una volta, grazie a un processo di deformazione metamorfica. Scrive la Rosselli: «E così saprai chi sono: la stupida ape che ronza / per un punto fermo, cercando Lui, quella giungla / di alberi di ferro battuto». (CoRTELLESSA 2007, 5). Si confronti l'immagine dell'ape con questa tratta da October Elizabethans nella descrizione di un rapporto amoroso non corrisposto: «I [...] do continue sending you Missiles; / Plunging my Prick into your Heart like a Bee» (Rosselli 2009, 59). La «stupida ape», quindi un'ape al femminile, dispone di un corpo androgino: nella poesia inglese la componente maschile viene esaltata esponenzialmente in una tensione fallica al quadrato, risultato di un fitto intreccio di rimandi linguistici e visivi (si pensi all'uso di parole a sfondo sessuale come «missiles» e «prick», che evocano esplicitamente il fallo, e all'immagine dell'ape che affonda nel cuore il suo pungiglione, ricalcando l'atto della penetrazione).

Il «Lui» ricercato è l'uomo nella veste di amante, è il Dio Padre ed è anche il padre in quanto genitore biologico. Il Lui, quindi la presenza maschile nel testo, si carica di referenti multipli in costante slittamento tanto da confondere l'autrice stessa: «Io non so cosa voglio tu non sai / chi sei, e siamo quasi pari» (Cortellessa 2007, 3). L'univocità del referente non può più esistere dal momento che la realtà è sottoposta ai «tanti filtri delle esperienze» (CORTELLESSA $2007,16)$; la confusione identitaria ritraccia il percorso che si era già verificato proprio in October Elizabethans, dove alla figura del padre assassinato si sovrapponeva quella dell'amante (incestuoso dunque) e quella dello stesso Dio. Il Figlio, Cristo, diventa per effetto metonimico la figlia che cerca l'unità nel Padre, il «Jesù che seppe / torcersi fuori dalla miseria, in tempo giusto / per la carne di Dio, per lo spirito di Dio» (CORTELLESSA 2007, 16).

Proprio questo Cristo emblematico racchiude il dramma dell'amoroso conflitto con i padri, che può essere letto come trauma primordiale, dell'infanzia, e allo stesso tempo come tentativo della sua risoluzione. Il Cristo, non per caso, rientra 
nella simbologia archetipica dell'androgino ${ }^{19}$ e quindi nel meccanismo di fusione e omogeneizzazione di ciò che si presenta come scisso ed eterogeneo. Il Jesù rosselliano si carica, dunque, di una serie di valori simbolici aggiunti in grado di fare luce su vari aspetti del rapporto dell'io-scrivente con la figura paterna. Jung non solo fa coincidere l'androginia del Cristo con l'individuazione del sé (Selbstwerdung), ma svela la natura incestuosa alla base dell'unione androgina:

Il contemporaneo "intervento" dello Spirito Santo però svela il senso riposto dell'incesto, dell'unione di fratello e sorella o di madre e figlio, simbolo audace di unio mystica. [...] L'incesto simboleggia l'unione col proprio essere, la individuazione o attuazione di sé (Selbstwerdung), e, data l'importanza vitale che riveste, esercita un fascino a volte quasi mostruoso, se non nella brutale realtà, quanto meno nell'evento psichico controllato dall'inconscio. (JUNG 1961, 65).

L’immagine di Cristo include in sé la compresenza degli opposti e dà vita a ciò che è «l'uomo primordiale di natura ermafrodita [...] l'uomo rotondo, cioè perfetto, dell'era primordiale e finale, inizio e fine dell'uomo» (JUNG 1961, 63). Il rapporto che sfocia nella «unio mystica», si riflette nella natura osmotica delle relazioni tra i referenti-personaggio: io, tu, lui, lei, sono parole-contenitori, vasi comunicanti, che si prestano a ricevere identità multiple:

Montale parla di Diana. Una bella dea. Come se l'avesse vista. C’è una separazione tra l'io e il tu che infastidisce quasi. Ne la Libellula in generale, e in questa variazione dall'Esterina di Montale, tento di abbattere la divisione tra un io scrivente e un tu immaginato. Ho voluto creare una piena identificazione dell'io scrivente, che è anche il tu a cui mi rivolgo, con il tu di Esterina, che diventa un altro io. La separazione netta tra un io e un tu, presente in Montale come in altri poeti, è forse una cosa tipica del linguaggio maschile. (PESCE 1998)

Lo slittamento metamorfico dei referenti contribuisce al determinarsi, lo abbiamo visto, di un io androgino, plurimo, complesso quindi universale, spogliato di ogni connotato univoco: «in tutto il mondo putrame, esiste / un solo io, esiste un solo tu» (CORTELLESSA 2007, 12).

Abbandonata dunque qualsiasi pretesa di significazione incontrovertibile, ogni nuova rilettura della Libellula coincide con una possibile risemantizzazione del testo, che si dimostra profondamente «mobile» e «distorto» a seconda dei «tanti filtri delle esperienze» (CORTELLESSA 2007, 16) che vengono attivati. 
La poesia presenta un lacerto di realtà, un campione, ${ }^{20}$ una trascrizione di stampo fotografico offerta nella sua complessità. Si potrebbe dire, con Guglielmi, che viene raggiunto uno stato di «illeggibilità, nel senso tradizionale, del prodotto artistico moderno, il quale non offre un "discorso sul mondo" o la possibilità di infiniti discorsi (quanti sono gli interpreti), ma piuttosto "esempi" di realtà, elaborati allo stato neutro» (GUGLIELMI, 1964, 48).

Da questo punto di vista, persino l'ermeneutica del testo rosselliano subisce, in un intricato gioco di specchi, lo stesso processo deformante messo in atto dall'autrice: l'ermeneuta, rinunciando al rilevamento di un senso incontestabile del testo, abbraccia la complessità caotica dei mezzi e del reale, e con essa la complessità del mondo di fuori. Siamo all'interno di un mutamento che chiama direttamente in causa il lettore: il fruitore deve infatti rispondere ai nuovi stimoli postmoderni di ricezione dell'opera che si configura, per citare Eco, come un sistema aperto:

La tendenza al disordine che caratterizza positivamente la poetica dell'apertura dovrà essere tendenza al disordine dominato, alla possibilità compresa in un campo, alla libertà sorvegliata da germi di formatività presenti nella forma che si offre aperta alle libere scelte del fruitore. (ECO 2006, 123; corsivo dell'autore)

Così Amelia Rosselli presenta al lettore, espone, un «disordine dominato» $\mathrm{e}$ «fecondo», nell'ottica di quel realismo deformante in grado di applicare trasformazioni su campionature di realtà (le esperienze fenomeniche) per proporre valori equivalenti, sempre aperti a più possibili ricezioni. 


\section{Note}

1 Se Fausto Curi si domanda, in modo piuttosto retorico: «Sarebbe azzardato, a questo punto, avanzare l'ipotesi che una parte almeno della giovane poesia italiana abbia una particolare radice fenomenologica?» (CURI 1965, 77), Anceschi riconosce direttamente l'influenza del pensiero fenomenologico, in particolare della scuola di Banfi, sulla produzione poetica del tempo: «Ma qui si dovrà parlare di poesia, e, ad indicare l'atmosfera in cui vivemmo, basterà ricordare ormai cosa fu la scuola di Antonio Banfi, per noi, un maestro, veramente, un uomo che lasciava alla verità tutta la sua flessibilità, ricchezza e movimento e non sembrava imporre nulla, anzi sollecitava in noi il nascere del nostro essere autentico» (ANCESCHI 1952, 12).

2 Amelia Rosselli viene a conoscenza del saggio di Charles Olson, scritto nel 1950 e intitolato Projective Verse, abbastanza presto, sicuramente prima della stesura di Spazi metrici del 1961 e con ogni probabilità in concomitanza con la stesura del Diario in Tre Lingue, che la Rosselli ricollega al problema avanzato proprio da Olson: «[I]l famoso problema di Olson, il campo se vuoi magnetico [...] DTL è interessante per alcuni perché ancora junghiano, ancora poundiano, ancora montaliano, ma c'è questo problema del campo magnetico. E fu scritto mi pare tra i 24 25 e 26 anni. Ma era interessante questo uscir di casa e osservare quello che ti capitava e scrivere allo stesso tempo, non proprio ciecamente [...] la partecipazione con l'ambiente tutt'altro che automatica, diretta: mentre vivi, cerchi di trascrivere le tue impressioni dell'ambiente, cosa ci fai dentro insomma». (DE MARCH e VENTURINI 2010, 205). Il manifesto di Charles Olson viene pubblicato per la prima volta nel 1950, sul terzo numero della rivista Poetry New York. In Italia arriva invece nel febbraio 1961, grazie alla traduzione di Aldo Tagliaferri sul primo numero del Verri di quell'anno.

Il rapporto tra Amelia Rosselli e Charles Olson è già stato osservato in altri testi critici a cui si rimanda: Stefano COLANGELO, Metrica come composizione, Bologna, Gedit, 2002; la sezione dedicata ad Amelia Rosselli redatta da Stefano Colangelo in Poesia del Novecento italiano, a c. di Niva Lorenzini, Roma, Carocci, 2002; e anche Paolo GIOvANNETTI e Gianfranca LAVEzZI, La metrica italiana contemporanea, Roma, Carocci, 2010.

3 Giuliani dichiara: «Così non abbiamo nessuna difficoltà a comprendere, in questo momento, $\mathrm{e}$ inserire nei nostri arnesi anche il tipo americano (di cui ci parla Charles Olson) di quel verso "dinamico" o "aperto" o "atonale" che abbiamo già sperimentato negli ultimi anni» (GIULIANI 1961, 190).

4 Giuliani scrive: «la visione "schizomorfa" con cui la poesia contemporanea prende possesso di sé e della vita presente» (GIULIANI 1972, XVIII).

5 Durante il ciclo di conferenze per celebrare i 40 anni dalla nascita del Gruppo 63 Niva Lorenzini introduce la figura di Amelia Rosselli in questo modo: «Amelia Rosselli non si lascia identificare con l'avanguardia, disposta com'è - sono avvertenze sue - a caricarsela semmai sulle spalle, e a rappresentarla mentre "sputa come una vecchia fattucchiera". Giudizio da decodificare, ovviamente; e però credo che, al di là di prese di posizione più o meno polemiche o bisognose di venire contestualizzate, la Rosselli abbia un suo posto importante in queste nostre giornate, dal momento che il suo linguaggio si può a buon diritto confrontare con le linee sperimentali che stanno emergendo nelle letture di questi giorni» (BARILLI, CURI e LORENZINI 2005, 66).

6 Si noti come questo termine rifletta, forse per altri motivi, una delle difficoltà di Amelia Rosselli di fronte alla propria malattia (le era stata diagnosticata la schizofrenia paranoide): "Quando nella visione "schizomorfa" individuavo il carattere fondamentalmente comune ai "novissimi" ero affascinato dalle ovvie implicazioni socio-culturali contenute nella definizione della schizofrenia come modalità dell'esistenza in cui sono altrettanto impossibili la soggettività e l'oggettività del mondo» (GIULIANI 1972, 9).

7 La citazione è tratta da un passaggio che mette a fuoco proprio la questione del riconoscimento della realtà come scopo dello scrivere. Di seguito è riportato l'intero paragrafo: «[I]l riconoscimento della realtà rimane lo scopo dello scrivere. Ma come potrà effettuarsi? La lingua che ha fin qui istituito rapporti di rappresentazione con la realtà [...] dovrà cambiare punto di vista. E cioè trasferirsi nel cuore della realtà, trasformandosi da specchio riflettente in accurato registratore di processi, anche i più irrazionali, del formarsi del reale; oppure, 
continuando a rimanere all'esterno della realtà, porre tra se stessa e questa un filtro attraverso il quale le cose, allargandosi in immagini surreali o allungandosi in forme allucinate, tornino a svelarsi. Questa è l'operazione essenziale del nuovo sperimentalismo» (BALESTRINI 2002, XIV).

8 La Rosselli, commentando la scelta del titolo, dichiara: «Il termine "serie" nel suo significato più semplice: cioè "serie" di poesie scritte pressappoco nello stesso periodo; vorrei con la parola "serie" dare carattere di neutralità all'alternarsi dei temi e delle forme (le poesie sono in stretto ordine cronologico). Però la parola "serie" può riferirsi anche alle "serie" matematiche, tendenti a zero a 1 e a x; si riferisce inoltre alle "serie" dodecafoniche e postdodecafoniche che hanno anch'esse carattere neutro e di inevitabilità» (CORTELLESSA 2007, 37).

9 Per approfondire il discorso si veda lo studio di Chiara Carpita intitolato Tre scritti e un acquerello per Ernst Bernhard (CORTELLESSA 2007, 129-135).

10 Per un'interessante analisi dell'androginia nell'opera di Amelia Rosselli e dell'identificazione dell'io con il padre defunto, si veda Altri corpi: poesia e corporalità negli anni Sessanta di Gian Maria Annovi. In particolare per l'integrazione del padre nell'io rosselliano: «Nel celebre saggio del 1923 L'To e l'Es, Sigmund Freud individua nel lutto la struttura incipiente della formazione dell'Io, una tesi che lo studioso aveva già abbozzato nel precedente Lutto $e$ melanconia (1917). Quando si perde un altro essere umano che si è amato, scrive Freud, l'Io incorpora l'altro nella sua struttura, assumendone gli attributi. È cioè attraverso un atto di identificazione che l'Io accoglie nella sua struttura l'oggetto perduto che si desiderava o si amava: «rifugiandosi nell'Io, l'amore si sottrae così alla dissoluzione». Questo tipo di identificazione non è occasionale ma si configura come una nuova struttura dell'identità: l'altro diventa in pratica parte dell'Io mediante l'interiorizzazione permanente dei suoi attributi» (ANNOVI 2008, 101).

11 In realtà la datazione della Libellula è oggetto di discussione, la Rosselli indica il 1958 come anno di stesura: data più che altro simbolica dal momento che un frammento del poemetto apparso sul Verri nel 1963 presenta differenze sostanziali rispetto alla versione ora in Poesie. A riguardo si veda il contributo di Stefano Giovannuzzi Come lavorava Amelia Rosselli, (CORTELLESSA 2007, 20-35).

12 Il richiamo fonetico tra Libellula e bellicosità (da bellum) è stato fatto notare, tra gli altri, da Gian Maria Annovi (ANNOvi 2008, 110) e da Theresia Prammer in Una Rosa è una rosa non è una rosa: riscrivere Amelia Rosselli (CORTELLESSA 2007, 107).

13 Amelia Rosselli, parlando della Libellula e della sua matrice femminista dirà: «È in parte un poemetto politico, femminista oltre che poetico. In Italia nel $1958 \mathrm{di}$ femminismo se ne parlava ben poco, ma io sono anche inglese e in Inghilterra il femminismo è sentito fin dalla fine del diciannovesimo secolo. Dunque mi era meno difficile assimilare certe tematiche in anticipo rispetto alle italiane» (CAMBON 1996, 71). Per una panoramica sulla scrittura femminile nel secondo Novecento si veda anche la tesi di dottorato di Ambra Zorat, La poesia femminile dagli anni Settanta a oggi. Percorsi di analisi testuale.

14 Emanuela Tandello sottolinea la centralità della figura della Fanciulla nell'opera rosselliana: una fanciulla-figlia che non è in grado di raggiungere piena maturazione in seguito al trauma reiterato del lutto. L'adolescenza, per la Rosselli, viene a identificarsi con una "stagione "unica" di perdita» (TANDELLO 2007, 7). La scrittura stessa si determina attraverso una visione adolescenziale del mondo, assumendo la forma di una struttura aperta, ovvero una «"struttura di crisi" di cui l'adolescente è l'incarnazione.» (TANDELLO 2007, 9).

15 Gian Maria ANNovi, Altri corpi: poesia e corporalità negli anni Sessanta, Bologna, Gedit, 2008; Rosaria Lo Russo, I Santi padri e la figlia dal cuore devastato, in La furia dei venti contrari: variazioni Amelia Rosselli con testi inediti e dispersi dell'autrice, a c. di Andrea Cortellessa, Firenze, Le lettere, 2007; Francesco CARBOGNIN, Le armoniose dissonanze: Spazio metrico e intertestualità nella poesia di Amelia Rosselli, Bologna, Gedit, 2008.

16 Le implicazioni di questa precisa scelta segnica vengono analizzate con estrema chiarezza in Altri corpi: poesia e corporalità negli anni Sessanta di Gian Maria Annovi: "Appare dunque ovvio che nel progetto di revisione del canone approntato da Amelia Rosselli nella Libellula avvenga anche un rovesciamento di genere: "io non so se tra le pallide rocce il tuo / sorriso m'apparve, o deo dalle fulgide chiome". Si noti come l'epiteto "deo", non solo non sia presente 
nell'originale campaniano ma anche come esso sia fortemente marcato dal punto di vista del genere. La Rosselli, infatti, non si limita ad impiegare il termine corrente "dio" ma varia, ancora una volta, sul femminile "dea", scegliendo di utilizzare una voce arcaica per rimarcare con maggior forza il cambio di desinenza. La scelta di trasformare un forte simbolo femminile come la Chimera in un tu maschile è, come si è detto, giustificata dal desiderio della poetessa di confrontarsi con il modello della tradizione, se non fosse che proprio tale tu rosselliano si rivela essere profondamente instabile dal punto di vista del genere». (ANNOVI 2008, 112).

17 Virginia Woolf infatti riporta: «Have you any notion of how many books are written about women in the course of a year? Have you any notion of how many are written by men? Are you aware that you are, perhaps, the most discussed animal in the universe?» (WoOLF 1988, 27), e poco dopo: «It was a most strange phenomenon; and apparently - here I consulted the letter M - one confined to the male sex. Women do not write books about men» (WoOlF 1988, 28).

18 Come ci fa notare John Berger: «One might simplify this by saying: men act and women appear. Men look at women. Women watch themselves being looked at. This determines not only most relations between men and women but also the relation of women to themselves. The surveyor of woman in herself is male: the surveyed female. Thus she turns herself into an object» (BERGER 2008, 47). Un'annotazione simile era già stata avanzata anche da Simone De Beauvoir: «Quando si specchiava ammirandosi, non faceva ancora che sognare: si sognava attraverso occhi di uomo; ora gli occhi sono presenti: impossibile barare» (DE BEAUVOIR 1984, 128).

19 Jung ne parla ne La psicologia del transfert: «La simbolica ecclesiastica di sponsus e sponsa porta all'unità mistica di entrambi, cioè all'anima Christi che vive nel corpus mysticum della Chiesa. Questa unità costituisce il fondamento dell'androginia di Cristo [...] un Cristo androgino, che da parte sua non dovrebbe mancare di un qualche rapporto interno con l'idea platonica dell'uomo primordiale androgino»» (JUNG 1961, 144).

20 A Birth si conclude con un'autodefinizione: la prosa è un «sample of recognition»; quindi esempio o campione di una presa di coscienza.

\section{Bibliografia}

\section{ANCESCHI 1952}

Linea lombarda: Sei poeti (Editi e inediti di Vittorio Sereni, Roberto Rebora, Giorgio Orelli, Nelo Risi, Renzo Modesti, Luciano Erba), a c. di Luciano Anceschi, Milano, Edizioni Magenta, 1952.

\section{ANNOVI 2008}

Gian Maria ANNOVI, Altri corpi: poesia e corporalità negli anni Sessanta, Bologna, Gedit, 2008.

\section{BACHTIN 1997}

Michail BACHTIN, Estetica e romanzo, Torino, Einaudi, 1997.

\section{BALESTRINI e GIULIANI 2002}

Gruppo '63. L'antologia, a c. di Nanni Balestrini, Alfredo Giuliani, Torino, Testo \& Immagine s.r.l, 2002.

\section{BARILLI, CURI e LORENZINI 2005}

Il gruppo '63 quarant'anni dopo: Bologna, 8-11 maggio 2003: atti del convegno, a c. di Renato Barilli, Fausto Curi, Niva Lorenzini, Bologna, Edizioni Pendragon, 2005, volume 1 . 
BARILLI e GUGLIELMI 2003

Gruppo 63. Critica e teoria, a c. di Renato Barilli, Angelo Guglielmi, Torino, Testo \& Immagine s.r.l, 2003.

BERGER 2008

John BERGER, Ways of Seeing, London, Penguin Books, 2008.

CAMBON 1996

Marina CAMBON, Incontro con Amelia Rosselli, in «DonnaWomanFemme», 1 (29) gennaio-marzo, 1996.

CAPUTO 2004

Una scrittura plurale: saggi e interventi critici - Amelia Rosselli, a c. di Francesca Caputo, Novara, interlinea, 2004.

CARBOGNIN 2008

Francesco CARBOGNIN, Le armoniose dissonanze: Spazio metrico e intertestualità nella poesia di Amelia Rosselli, Bologna, Gedit, 2008.

COLANGELO 2002

Stefano ColANGELO, Metrica come composizione, Bologna, Gedit, 2002.

CORTELLESSA 2007

La furia dei venti contrari: variazioni Amelia Rosselli con testi inediti e dispersi dell'autrice, a c. di Andrea Cortellessa, Firenze, Le lettere, 2007.

COURANT e ROBBINS 1964

Richard COURANT e Herbert ROBBINS, Che cos'è la matematica? Introduzione elementare ai suoi concetti e metodi, Torino, P. Boringhieri, 1964.

CURI 1965

Fausto CURI, Ordine e disordine, Milano, Feltrinelli, 1965.

DE BEAUVOIR 1984

Simone DE BEAUVOIR, Il secondo sesso, Milano, il Saggiatore, 1984.

DE MARCH e VENTURINI 2010

È vostra la vita che ho perso: conversazioni e interviste, 1964 - 1995 - Amelia Rosselli, a c. di Silvia De March e Monica Venturini, Firenze, Le lettere, 2010.

FRABOTTA 1976

Donne in poesia. Antologia della poesia femminile in Italia dal dopoguerra a oggi, a c. di Biancamaria Frabotta, Roma, Savelli, 1976.

GIOVANNETTI e LAVEZZI 2010

Paolo GiovanNETTI e Gianfranca LAVEZZI, La metrica italiana contemporanea, Roma, Carocci, 2010. 


\section{GIULIANI 1961}

I Novissimi: poesie per gli anni '6o, a c. di Alfredo Giuliani, Milano, Rusconi e Paolazzi editori, 1961.

GUGLIELMI 1964

Angelo GUGLIELMI, Avanguardia e sperimentalismo, Milano, Feltrinelli, 1964.

LORENZINI 2002

Poesia del Novecento italiano, a c. di Niva Lorenzini, Roma, Carocci, 2002.

LOTMAN 1990

Jurij LOTMAN, La struttura del testo poetico, Milano, Mursia, 1990.

MENGALDO 1994

Pier Vincenzo Mengaldo, Poeti italiani del Novecento, Milano, Mondadori, 1994.

OLSON 1966 [1950]

Charles OLSON, Selected writings / edited, with an introduction by Robert Creeley, New York, New Directions Publishing, 1966.

OLSON 1950

Charles OLSON, Projective Verse, in «Poetry New York», n.3, 1950.

OLSON 1961

Charles Olson, Il verso proiettivo, tradotto da Aldo Tagliaferri, in «Verri», n.1, 1961.

PESCE 1998

Ulderico PESCE, La donna che vola, in Amelia Rosselli. Un'apolide alla ricerca del linguaggio universale, Atti della giornata di studio a Firenze, Gabinetto Viesseux, 29 Maggio 1998, «Quaderni del Circolo Rosselli», n. 17.

ROSSELLI 2009

Amelia Rosselli, Le Poesie, Milano, Garzanti, 2009.

SHOWALTER 1986

The New Feminist Criticism, a c. di Elaine Showalter, Londra, Virago Press, 1986.

TANDELLO 2007

Emanuela TANDELlo, Amelia Rosselli. La fanciulla e l'infinito, Roma, Donzelli editore, 2007.

WOOLF 1988

Virginia WoOLF, A Room of One’s Own, London, Grafton Books, 1988.

ZORAT 2009

Ambra ZoRAT, La poesia femminile dagli anni Settanta a oggi. Percorsi di analisi testuale, 2009, consultato all'indirizzo <http://www.openstarts.units.it/dspace/bitstream/10077/3771/4/Zorat_phd.pdf> (20.03.2014). 